\title{
IMUNIZAÇÃO DO RISCO DE TAXA DE JUROS DE MERCADO (ETTJ) EM UMA CARTEIRA PREVIDENCIÁRIA SIMULADA
}

\author{
Thierry Faria da Silva Gregorio \\ Universidade do Estado do Rio de Janeiro e Centro de Análises de Sistemas Navais \\ e-mail: thierrygregorio@ime.uerj.br \\ Guilherme Dornelas Vianna \\ IRB-Brasil Resseguros \\ e-mail: guilherme-dornelas@ hotmail.com \\ João Santoro D’Amato \\ MRS Global \\ e-mail: $\underline{\text { santoro.joao@gmail.com }}$ \\ Marcos dos Santos \\ Centro de Análises de Sistemas Navais \\ e-mail: marcosdossantos_coppe_ufrj@yahoo.com.br
}

\section{RESUMO}

Nesta pesquisa apresentam-se as principais estratégias de imunização do risco da taxa de juros de mercado (ETTJ), utilizando medidas clássicas na avaliação de riscos, a duration (duração), a duração modificada e a convexidade. O objetivo deste estudo é realizar a imunização do risco de taxa de juros do passivo de uma carteira previdenciária simulada de uma Entidade de Previdência Complementar, na qual os recursos garantidores são investidos em títulos públicos pós-fixados em IPCA, denominados Notas do Tesouro Nacional - Série B (NTN-B). A principal linha de ação é igualar a duração modificada do ativo em relação ao passivo no intuito de alinhar os valores presentes do ativo e do passivo quando haja alterações nas taxas de juros de mercado.

Palavras-Chaves: Imunização, Risco de Taxa de Juros, Duração Modificada e Estrutura a Termo de Taxa de Juros

\begin{abstract}
This research presents the main strategies of immunization of the interest rate risk in the financial market, using classical measures of the risk assessment, like duration, the modified duration and convexity. The aim of this study is to perform the immunization of the interest rate risk of the liability from simulated portfolio of a pension fund entity which the guarantor resources are invested in bonds pos fixed in IPCA called National Treasury Notes series B (NTN - B). The target of this study is match the modified duration of the asset with the modified duration of the liability, in order to align the present values of assets and liabilities when there are changes in the market interest rate.
\end{abstract}

Keywords: Immunization, Interest Rate Risk, Modified Duration, Term Structure of Interest Rate 


\section{Introdução}

Em uma economia dinâmica e globalizada o ambiente financeiro é cada dia mais imprevisível, como demonstram as recentes crises financeiras internacionais. No intuito de criar administrações financeiras eficientes para esse mercado volátil, os gestores de risco buscam constantemente criar instrumentos que possam auxiliar na redução do risco de mercado, em especial o risco de variação das taxas de juros.

A análise do risco de taxa de juros, dentre outros riscos, é de grande importância na administração dos ativos e passivos (Asset Liability Management ALM) de um passivo previdenciário e de uma carteira de renda fixa, pois busca diminuir ou até eliminar os impactos das variações nas taxas de juros de mercado no valor presente do ativo e do passivo, minimizando possíveis prejuízos para a entidade, participantes e beneficiários.

Um dos principais conceitos de gerenciamento do risco de taxa de juros é o conceito de imunização, que tem como foco eliminar o efeito econômico das variações da taxa de juros sobre os valores presentes de determinada carteira. A imunização clássica segundo Redington (1952) consiste em manter a igualdade entre o valor presente do ativo e o valor presente do passivo frente a variações futuras nas taxas de juros.

Uma das estratégias mais utilizadas para a avaliação da exposição de uma carteira ao risco de variação de taxa de juros é a duração modificada. A duração modificada de acordo com Bierwag (1977) avalia a sensibilidade do valor atual de um ativo ou de um passivo em relação às variações nas taxas de juros, o que pode ser interpretado matematicamente como a primeira derivada da função valor presente dos fluxos financeiros em relação à taxa de juros. Esta estratégia considera que as variações nas taxas de juros são paralelas, ou seja, ocorrem simultaneamente e na mesma proporção em todos os vencimentos.

Além da duração modificada, pode-se trabalhar com a convexidade (Bierwag,1977) que representa a segunda derivada da função valor presente dos fluxos financeiros em relação à taxa de juros, permitindo desta forma minimizar o erro de aproximação entre o valor presente estimado, utilizando a duração modificada e a convexidade, e o verdadeiro valor presente.

O mercado financeiro habitualmente adota a duração instituída por Macaulay (1938) que considera que as taxas de juros são constantes para todos os horizontes temporais e a duração instituída por Fisher e Weil (1971) que considera a Estrutura a Termo de Taxa de juros como fator de desconto financeiro.

Neste estudo abordaremos uma estratégia imunizadora na qual considera a Estrutura a Termo de Taxa de Juros como fator de desconto, visto que para fins de solvência é necessário avaliar os direitos e obrigações a valor de mercado (Gregorio, 2013).

\section{Asset Liability Management - ALM}

De forma abrangente podemos definir a estratégia de ALM, como o processo de monitoramento das operações financeiras, no intuito de assegurar que os objetivos e interesses dos investidores, participantes e beneficiários sejam alcançados e que o valor de seus ativos ou investimentos sejam maximizados. Os principais objetivos desse tipo de gestão consistem em garantir que os recursos garantidores alcancem os 
rendimentos necessários para fazer jus às obrigações da entidade com os participantes e segurados.

O ALM tem como principal foco a gestão dinâmica da carteira, focando inicialmente, nos riscos de taxas de juros e na forma a qual estes riscos podem impactar no balanço contábil, sem deixar de avaliar os riscos de crédito, de liquidez e de subscrição. Na atuária, as idades dos participantes, as tábuas de mortalidade e a rotatividade ajudam a projetar o fluxo financeiro do passivo, o qual deve estar garantido através dos investimentos materializados no ativo. A gestão das obrigações da entidade frente aos participantes e segurados e a administração dos recursos garantidores das provisões matemáticas são grandes desafios para os atuários.

No caso das seguradoras e entidades de previdência complementar, ou seja, carteiras com passivos atuariais, a gestão integrada dos ativos e passivos com o objetivo de minimizar o risco de descasamento entre eles virou um item indispensável em qualquer estratégia de gestão de riscos financeiros. Devido a esse tratamento integrado de ativos e passivos, visando alocar os ativos de acordo com a necessidade vinda do passivo, instituiu-se o conceito de ALM.

Segundo De La Peña (2008) podem-se classificar as estratégias de ALM em congruência por durações, congruência temporal ou congruência absoluta. A congruência por durações consiste em igualar as durações modificadas do ativo e do passivo com o intuito de imunizar o balance financeiro em relação a mudanças nas taxas de juros. A congruência temporal tem por objetivo realizar o casamento dos fluxos de caixa do ativo em relação ao passivo durante um período determinado e igualar as durações modificadas. Já a congruência absoluta se baseia em realizar o casamento de todos os fluxos de caixa do ativo em relação ao passivo.

\section{Duração, Duração Modificada e Convexidade de Macaulay}

\subsection{Duração}

O conceito de duração foi introduzido pela primeira vez por Macaulay (1938) que é basicamente o comprimento de um título ou, em termos estatísticos, o vencimento médio dos fluxos de caixa. A teoria de Macaulay considerou cada fluxo financeiro (cupom ou principal) como um título zero cupom que tem como fator de desconto uma taxa de juros constante, desta forma o somatório dos diversos títulos zero cupom com diferentes valores e maturidades corresponderia ao título original.

A continuação se expressa a duração instituída por Macaulay:

$$
\text { Duração }=\sum_{t=1}^{N} \frac{t \cdot C_{t} \cdot(1+r)^{-t}}{C_{t} \cdot(1+r)^{-t}}=\sum_{t=1}^{N} \frac{t \cdot C_{t} \cdot(1+r)^{-t}}{V P}
$$

$C_{t}=$ Fluxo de Caixa no tempot

$t=$ Prazo até $o$ vencimento em dias úteis

$r=$ taxa de juros constante para todos os vencimentos

$V P=$ Valor Presente dos fluxos financeiros 
Enquanto a maturidade leva em consideração apenas o prazo até o vencimento do principal, a duração, como visto na expressão (1), calcula o tempo médio de recebimento/pagamento dos fluxos de caixa ponderando cada instante $t$ pela proporção $\frac{C_{t} \cdot(1+r)^{-t}}{V P}$ que logicamente está compreendida no intervalo [0,1]. Portanto, considera-se a duração uma medida financeira mais adequada que a maturidade no que tange à expectativa de recebimento/pagamento dos fluxos financeiros futuros.

\subsection{Duração Modificada}

De acordo com Macaulay (1938) a duração modificada é oriunda do conceito matemático da derivada, visto que a sua formulação matemática é bastante semelhante à primeira derivada da função valor presente dos fluxos financeiros em relação à taxa de juros.

A função valor atual dos fluxos financeiros e a sua primeira derivada em relação à taxa de juros estão demonstradas respectivamente nas expressões (2) e (3).

$$
\begin{gathered}
V P=\sum_{t=1}^{N} C_{t} \cdot(1+r)^{-t} \\
\frac{\partial V P}{\partial r}=-\sum_{t=1}^{N} t \cdot C_{t} \cdot(1+r)^{-t-1}
\end{gathered}
$$

Como na duração de Macaulay as taxas de juros são constantes para os distintos vencimentos dos fluxos de caixa, é possível instituir a seguinte relação entre as expressões (3) e (1):

$$
\frac{\partial V P}{\partial r}=\frac{-D u r a c ̧ a ̃ o \cdot V P}{(1+r)}
$$

Portanto, instituiu-se a Duração Modificada como sendo a taxa de variação percentual do valor presente dos fluxos financeiros em relação a mudanças na taxa de juros de mercado $r$, como se exemplifica a continuação:

$$
\text { Duração Modificada }=\frac{\text { Duração }}{(1+r)}
$$

A utilização da Duração Modificada para estimar o valor presente dos fluxos financeiros é apropriada quando haja pequenas alterações nas taxas de juros de mercado, visto que estabelece uma relação linear entre as variações na taxa de juros e o valor presente, quando na prática esta relação é convexa. A seguir se estabelece a aproximação do valor presente pela duração modificada:

$$
\text { Novo } V P=V P+\frac{\partial V P}{\partial r} \cdot \Delta r=V P-\text { duração modificada } \cdot V P \cdot \Delta r
$$

$\Delta r=$ variação na taxa de juros de mercado 


\subsection{Convexidade}

As variações nas taxas de juros afetam o valor presente dos fluxos financeiros de uma forma não linear, ou seja, de uma forma convexa, desta forma quanto maior seja a taxa de juros de mercado, menor o valor presente da carteira. $O$ grau de convexidade pode ser maior ou menor, dependendo principalmente da dispersão dos vencimentos dos fluxos financeiros em relação à duração da carteira e da taxa de juros de mercado aplicada.

A convexidade é a segunda derivada do valor presente dos fluxos financeiros em relação à taxa de juros de mercado e descreve a maneira como a duração modificada varia em relação a mudanças nas taxas de juros de mercado. Logo, através da convexidade conseguimos diminuir o erro de aproximação ao estimar o valor presente dos fluxos financeiros aplicando a Série de Taylor.

$$
\text { Convexidade }=\frac{\partial^{2} V P}{\partial r^{2}}=\sum_{t=1}^{N} \mathrm{t} \cdot(\mathrm{t}+1) \cdot C_{t} \cdot(1+r)^{-t-2}
$$

Através da Série de Taylor se estima o valor presente utilizando a Duração Modificada e a Convexidade, como se expõe a seguir:

$$
\begin{gathered}
\text { Novo } V P=V P+\frac{\partial V P}{\partial r} \cdot \Delta r+\frac{1}{2} \cdot \frac{\partial^{2} V P}{\partial r^{2}} \cdot \Delta r^{2} \quad \therefore \\
\text { Novo } V P=V P-\text { duração modificada } \cdot V P \cdot \Delta r+\frac{1}{2} \cdot \text { Convexidade } \cdot \Delta r^{2}
\end{gathered}
$$

\section{Duração, Duração Modificada e Convexidade de Fisher e Weil}

\subsection{Duração}

O conceito de duração concebido por Fisher e Weil (1971) é semelhante ao estabelecido por Macaulay (1938), entretanto contempla a utilização de taxas de juros de mercado variáveis para trazer a valor presente os fluxos financeiros, permitindo assim calcular o valor presente dos fluxos financeiros utilizando a Estrutura a Termo de Taxa de Juros.

$$
\text { Duração }=\sum_{t=1}^{N} \frac{t \cdot C_{t} \cdot\left(1+r_{t}\right)^{-t}}{C_{t} \cdot\left(1+r_{t}\right)^{-t}}=\sum_{t=1}^{N} \frac{t \cdot C_{t} \cdot\left(1+r_{t}\right)^{-t}}{V P}
$$

$r_{t}=$ taxa de juros spot referente ao vencimento $t$

\subsection{Duração Modificada}

A duração modificada calculada em base a fluxos financeiros nos quais as taxas de juros de mercado variam de acordo com os vencimentos, segue o mesmo raciocínio matemático que a duração modificada em base a taxas de juros constantes. Portanto, a seguir se exemplifica a primeira derivada da função valor presente dos fluxos financeiros (considerando taxas de juros variáveis) em relação às taxas de juros:

$$
\frac{\partial V P}{\partial r}=-\sum_{t=1}^{N} t \cdot C_{t} \cdot\left(1+r_{t}\right)^{-t-1}
$$

Consequentemente, a duração modificada em base a teoria de Fisher e Weil (1971) segue a continuação: 


$$
\text { Duração Modificada }=\sum_{t=1}^{N} \frac{t \cdot C_{t} \cdot\left(1+r_{t}\right)^{-t}}{\left(1+r_{t}\right)}
$$

Neste caso em que se utilizam taxas de desconto variáveis, não é possível estabelecer uma relação matemática trivial entre a duração e a duração modificada como realizado na expressão (5) para taxas de desconto constantes. Concluindo, através da expressão (6) se estima o valor presente dos fluxos financeiros.

\subsection{Convexidade}

A convexidade de acordo com as premissas de taxas de desconto variáveis é dada pela segunda derivada da função valor presente dos fluxos financeiros e se expressa a seguir:

$$
\text { Convexidade }=\frac{\partial^{2} V P}{\partial r^{2}}=\sum_{t=1}^{N} \mathrm{t} \cdot(\mathrm{t}+1) \cdot C_{t} \cdot\left(1+r_{t}\right)^{-t-2}
$$

Utilizando a expressão (8) logra-se estimar o valor presente dos fluxos financeiros futuros caso haja alterações paralelas na Estrutura a Termo de Taxa de Juros, ou seja, considerando que as taxas de juros variam da mesma forma em todos os vencimentos.

\section{Estrutura do Passivo Previdenciário}

\subsection{Construção Da Massa De Participantes Do Passivo Previdenciário}

Para a construção do passivo previdenciário desta entidade de previdência hipotética, decidiu-se trabalhar com uma carteira composta por 1.000 participantes que estavam em condições de elegibilidade no ano de 2014 e deram entrada no pedido de aposentadoria programada. A idade de elegibilidade deste plano é de 55 anos. Portanto, a distribuição de novos assistidos em cada idade seguiu uma inversa uniforme, com parâmetros 55 e 65, gerada a partir de uma função aleatória que simulava as probabilidades entre 0 e 1 . A distribuição de idades simulada segue a continuação:

\begin{tabular}{ccc}
\hline Idades & Quantidade & Frequência \\
\hline 55 & 98 & $9,80 \%$ \\
56 & 91 & $9,10 \%$ \\
57 & 96 & $9,60 \%$ \\
58 & 92 & $9,20 \%$ \\
59 & 79 & $7,90 \%$ \\
60 & 89 & $8,90 \%$ \\
61 & 90 & $9,00 \%$ \\
62 & 89 & $8,90 \%$ \\
63 & 107 & $10,70 \%$ \\
64 & 82 & $8,20 \%$ \\
65 & 87 & $8,70 \%$ \\
\hline Total= & 1.000 & $100,00 \%$ \\
\hline
\end{tabular}

Tabela 1. Distribuição de idades simulada 
Para simular o valor dos benefícios mensais a serem percebidos pelos assistidos, foi utilizada uma inversa normal com os parâmetros média $=\mathrm{R} \$ 3.966,00$ que é a média dos benefícios dos fundos de pensão brasileiros segundo o Periódico Fundos de Pensão (2013) e desvio-padrão= $\mathrm{R} \$ 396,60$ que foram gerados a partir de uma função aleatória que simulava as probabilidades entre 0 e 1 .

\subsection{Cálculos do Passivo Previdenciário}

A tábua utilizada para projetar os fluxos financeiros futuros dos participantes assistidos foi a BR-EMSsb-v.2010-m que foi criada pela Universidade Federal do Rio de Janeiro em base a experiência do mercado segurador brasileiro.

Para o cálculo do valor presente da carteira, foi utilizada a Estrutura a Termo de Taxa de Juros em base ao modelo de Svensson (1994) disponibilizada pela Superintendência de Seguros Privados (2014), desta forma foi possível projetar as taxas spot para todos os vencimentos futuros nos quais a entidade de previdência tem obrigações junto aos participantes assistidos.

A ETTJ utilizada foi a calculada em base aos títulos públicos NTN-B, visto que os benefícios previdenciários desta carteira serão reajustados pela inflação do IPCA. Cabe destacar que a ETTJ proporcionada pelos títulos públicos NTN-B está formada por taxas spot reais.

\begin{tabular}{|c|c|c|c|c|c|c|c|c|c|c|c|}
\hline $\begin{array}{l}\text { Maturidade } \\
\text { (anos) }\end{array}$ & Taxa & $\begin{array}{c}\text { Maturidade } \\
\text { (anos) }\end{array}$ & Taxa & $\begin{array}{c}\text { Maturidade } \\
\text { (anos) }\end{array}$ & Taxa & $\begin{array}{c}\text { Maturidade } \\
\text { (anos) }\end{array}$ & Taxa & $\begin{array}{c}\text { Maturidade } \\
\text { (anos) }\end{array}$ & Taxa & $\begin{array}{c}\text { Maturidade } \\
\text { (anos) }\end{array}$ & Taxa \\
\hline 1 & $4,33 \%$ & 13 & $6,63 \%$ & 25 & $6,86 \%$ & 37 & $6,95 \%$ & 49 & $7,00 \%$ & 61 & $7,03 \%$ \\
\hline 2 & $5,51 \%$ & 14 & $6,66 \%$ & 26 & $6,87 \%$ & 38 & $6,96 \%$ & 50 & $7,01 \%$ & 62 & $7,03 \%$ \\
\hline 3 & $5,90 \%$ & 15 & $6,69 \%$ & 27 & $6,88 \%$ & 39 & $6,97 \%$ & 51 & $7,01 \%$ & 63 & $7,04 \%$ \\
\hline 4 & $6,10 \%$ & 16 & $6,71 \%$ & 28 & $6,89 \%$ & 40 & $6,97 \%$ & 52 & $7,01 \%$ & 64 & $7,04 \%$ \\
\hline 5 & $6,22 \%$ & 17 & $6,74 \%$ & 29 & $6,90 \%$ & 41 & $6,97 \%$ & 53 & $7,01 \%$ & 65 & $7,04 \%$ \\
\hline 6 & $6,30 \%$ & 18 & $6,76 \%$ & 30 & $6,91 \%$ & 42 & $6,98 \%$ & 54 & $7,02 \%$ & 66 & $7,04 \%$ \\
\hline 7 & $6,37 \%$ & 19 & $6,78 \%$ & 31 & $6,92 \%$ & 43 & $6,98 \%$ & 55 & $7,02 \%$ & 67 & $7,04 \%$ \\
\hline 8 & $6,43 \%$ & 20 & $6,79 \%$ & 32 & $6,92 \%$ & 44 & $6,99 \%$ & 56 & $7,02 \%$ & 68 & $7,04 \%$ \\
\hline 9 & $6,48 \%$ & 21 & $6,81 \%$ & 33 & $6,93 \%$ & 45 & $6,99 \%$ & 57 & $7,02 \%$ & 69 & $7,05 \%$ \\
\hline 10 & $6,52 \%$ & 22 & $6,82 \%$ & 34 & $6,94 \%$ & 46 & $6,99 \%$ & 58 & $7,03 \%$ & 70 & $7,05 \%$ \\
\hline 11 & $6,56 \%$ & 23 & $6,84 \%$ & 35 & $6,94 \%$ & 47 & $7,00 \%$ & 59 & $7,03 \%$ & 71 & $7,05 \%$ \\
\hline 12 & $6,60 \%$ & 24 & $6,85 \%$ & 36 & $6,95 \%$ & 48 & $7,00 \%$ & 60 & $7,03 \%$ & & \\
\hline
\end{tabular}

Tabela 2. Estrutura a Termo de Taxa de Juros (Data Base 31/03/2014)

Para calcular o valor presente de cada participante assistido, foi utilizada a seguinte metodologia:

$$
\mathrm{VP}=\sum_{t=1}^{N} \frac{C_{t} \text { projetado }}{\left(1+r_{t}\right)^{\mathrm{t}}}
$$

$C_{t}$ projetado $=13 \cdot$ benefício mensal $\cdot{ }_{t} p_{x}$

${ }_{t} p_{x}=$ probabilidade de que uma pessoa de idade $x$ chegue vivo à idade $x+$ t em base à tábua de mortalidade BR - EMSsb - v 2010-m 
A continuação se apresentam os principais resultados desta carteira:

- O valor presente calculado utilizando a fórmula (13) foi de R $\$ 576.356 .705,91$;

- A duração do passivo previdenciário foi calculada de acordo com a expressão (9) e resultou em 9,83 anos;

- Utilizando a expressão (11) foi calculada a duração modificada da carteira que foi de 9,22;

- Em base a expressão (12) a convexidade foi de 73.295.558.906,69.

\section{Estrutura do Ativo Previdenciário (Recursos Garantidores)}

\subsection{Composição da Carteira do Ativo}

O ativo desta entidade previdenciária estará composto basicamente pelos títulos públicos NTN-B que são pós-fixados em IPCA, os quais foram escolhidos porque os benefícios concedidos aos participantes assistidos serão reajustados ao longo do tempo de acordo com este índice de inflação. A entidade previdenciária dispõe de $\mathrm{R} \$ 576.356 .705,91$ para investir em títulos públicos, visto que este é o valor presente das suas obrigações frente aos participantes e este é o valor que corresponde aos recursos garantidores. A seguir se ilustram as possíveis NTN-B que podem ser negociadas no mercado secundário:

\begin{tabular}{c}
\hline Vencimentos NTN-B \\
\hline $15 / 05 / 2015$ \\
$15 / 05 / 2017$ \\
$15 / 08 / 2020$ \\
$15 / 08 / 2024$ \\
$15 / 05 / 2035$ \\
$15 / 05 / 2045$ \\
$15 / 08 / 2050$ \\
\hline
\end{tabular}

Tabela 3. Vencimentos disponíveis de NTN-B

Para a composição dos recursos garantidores do passivo desta entidade de previdência foram selecionados os títulos NTN-B com vencimento em 15/05/2015 e 15/08/2050. A metodologia utilizada para a adoção destes títulos foi à busca por estabelecer um ativo que possuísse a maior dispersão possível a fim de obter a maior convexidade. Cabe destacar que em 31/03/2013 o Valor Nominal Atualizado (VNA) das NTN-B tinha o valor de $\mathrm{R} \$ 2.263,83$, portanto os fluxos projetados de cupons semestrais têm o valor de $\mathrm{R} \$ 66,92$, pois a NTN-B paga cupons semestrais correspondentes a $6 \%$ anual do VNA.

\subsection{Cálculos do Ativo}

Como a Estrutura a Termo de Taxa de Juros formada pelos títulos públicos NTN-B é estimada através das Taxas Internas de Retorno (TIR) destes títulos, pode-se calcular o valor presente dos títulos desta carteira utilizando as TIR que se equivale a utilizar a ETTJ. Desta forma, calcula-se o valor presente, a duração modificada e a convexidade das NTN-B desta carteira em base as expressões (2), (5) e (7) respectivamente.

O título que vence em 15/05/2015 possui uma Taxa Interna de Retorno de $4,53 \%$, logo, obteve-se os seguintes resultados: 
- O valor presente deste título foi de $\mathrm{R} \$ 2349,30$;

- A duração modificada calculada foi de 1,03;

- Esta NTN-B apresenta a convexidade de 5156,15.

A segunda NTN-B elegida que vence em 15/08/2050 tem uma TIR de 6,67\%. A continuação se expõe os seus respectivos resultados:

- O valor atual deste ativo é R\$2.074,70;

- A duração modificada desta NTN-B é 13,47 ;

- A convexidade é 4.716.834,45.

\section{Conclusão e Imunização}

O principal objetivo deste trabalho é conseguir estabelecer a imunização do risco de taxa de juros no balance financeiro desta entidade previdenciária fictícia, logo, busca-se igualar a duração modificada do ativo em relação ao passivo. A continuação se expressa a metodologia para igualar a duração modificada do ativo ao do passivo:

$$
\begin{gathered}
\mathrm{DMp}=D M_{15} \cdot f_{15}+D M_{50} \cdot f_{50} \\
9,21=1,033 \cdot f_{15}+13,47 \cdot\left(1-f_{15}\right) \therefore \\
f_{15}=34,21 \% \\
e
\end{gathered}
$$

$D M p=$ Duração Modificada do Passivo

$D M_{15}=$ Duração Modificada do Título que vence em 2015

$D M_{50}=$ Duração Modificada do Título que vence em 2050

$f_{15}=$ Proporção de investimento no Título que vence em 2015

$f_{50}=$ Proporção de investimento no Título que vence em 2050

Portanto, ao investir 34,21\% ( $\mathrm{R} \$ 197.213 .841,39)$ do valor presente do passivo na NTN-B que vence em 2015 e 65,79\% (R\$ 379.142.864,52) na NTN-B que vence em 2050, iguala-se a duração modificada do ativo $(9,21)$ com a do passivo, estando assim imune ao risco de taxa de juros.

Além disto, estas proporções de investimentos proporcionam uma convexidade ao ativo de 862.413.267.152,90 que é bem superior a do passivo (73.295.558.906,69). Cabe destacar que quando a convexidade do ativo é superior a do passivo, o balance financeiro de uma entidade fica totalmente imune às variações de taxas de juros de mercado, pois a curvatura (convexidade) da função valor presente dos fluxos financeiros do ativo será sempre superior a do passivo. Portanto, para qualquer alteração na taxa de juros de mercado o valor atual dos recursos garantidores será superior ao valor atual das obrigações da entidade previdenciária frente aos participantes assistidos. 


\section{Referências Bibliográficas}

Associação Brasileira das Entidades Fechadas de Previdência Complementar. (2014). Consolidado Estatístico. Revista Fundos de Pensão, nº 390, São Paulo: 2014

Bierwag, G. O. (1977). Immunization, duration, and the term structure of interest rates. Journal of Financial and Quantitative Analysis. December, 1.977. Pp-725742.

Bierwag, G. O.; Kaufman, G. G. y Toevs, A. (1983). Duration: Its Development and Use in Bond Portfolio Management. Financial Analyst Journal. July-August, 1983. Pp.15-35

Conde, Cezar Newton. Tábua de Mortalidade Destinada a Entidades Fechadas de Previdência Privada. 1991. Tese de Mestrado. (Mestrado em Ciências Atuariais). Pontifícia Universidade Católica de São Paulo, São Paulo: 1991.

De la Peña, J. I. (1997). El riesgo de interés en seguros y pensiones: Una aproximación actuarial. Anales del Instituto de Actuarios Españoles, 1.996, Tercera Época, Número 2. Pp.49-172

De la Peña, J. I.; Plazaola, I.I. (2008). El problema del rebalanceo en las estrategias inmunizadoras. Análisis Financiero, 2008, Número 108. Pp.66-76

Fabozzi, F. J.; Modigliani, F. y Ferri, M. G. (1996). Mercados e Instituciones Financieras. Prentice - Hall Hispanoamericana S.A. (México)

Fisher, L. y Weil, R. (1971). Coping with the Risk of Interest-Rate Fluctuations: Returns to Bondholders from Naive and Optimal Strategies. Journal of Business, October, 1.971. Pp.408-431.

Gregorio, Thierry Faria da Silva (2013). Aplicación del modelo Smith-Wilson a la estructura temporal de tipos de Interés. Efectos sobre las provisiones matemáticas de una entidad aseguradora del ramo de vida. Tese de Mestrado. Facultad de Ciencias Economicas y Empresariales. Universidad de Málaga, Málaga: 2013

Mantovanini, Rosaura E. Morganti (1995). Macaulay Duration: Método para administrar o Risco de Taxa de Juros em Bonds sem Opção de Recompra e Isentas do Risco de Inadimplência. Tese de Mestrado. Fundação Getúlio Vargas de São Paulo, São Paulo: 1995.

Marques, Demósthenes (2011). Asset Liability Management (ALM) para entidades fechadas de Previdência complementar no Brasil. Tese de Mestrado. Universidade de Brasilia, Brasilia: 2011

Perez, José Maurício (1994). O Risco da Taxa de Juros em Carteira de Renda Fixa: Estratégias de Hedge no Mercado Futuro de Depósitos Interfinanceiros. Tese de Mestrado. Universidade Federal do Rio de Janeiro, Rio de Janeiro: 1994.

Redington, F.M. (1952). Review of the principles of Life-Office Valuations. The Journal of the Institute of Actuaries, $n^{\circ}$ 78, 1.952, London. pp- 286 - 340

Reitano, R. R. (1994). Non-Parallel Yield Curve Shifts and Immunization. Proceedings of the 4th AFIR, April 20-22, 1.994. .Orlando, Florida. Pp- 427 - 455 
Superintendência de Seguros Privados. (2014). Modelos de Interpolação e Extrapolação da ETTJ. Disponível em: www.susep.gov.br

Svensson, L. E. O. (1994). Estimating and Interpreting Forward Interest Rates: Sweden 1992 - 1994 , International Monetary Fund Working Paper, No. 114, Washington DC 